\title{
Design and Analysis of a Micromechanical Three-Component Force Sensor for Characterizing and Quantifying Surface Roughness
}

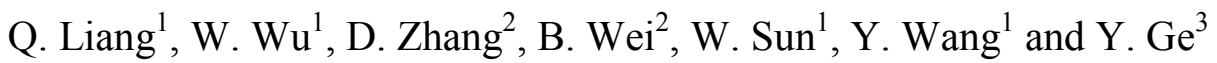 \\ ${ }^{1}$ College of Electrical and Information Engineering, Hunan University, Changsha, Hunan, 410082, China \\ ${ }^{2}$ Faculty of Engineering and Applied Science, University of Ontario Institute of Technology, Oshawa, ON L1H 7K4, Canada \\ ${ }^{3}$ State Key Laboratories of Transducer Technology, Institute of Intelligent Machines, Chinese Academy of Sciences, Hefei,
}

Anhui 230031, China

\begin{abstract}
Roughness, which can represent the trade-off between manufacturing cost and performance of mechanical components, is a critical predictor of cracks, corrosion and fatigue damage. In order to measure polished or super-finished surfaces, a novel touch probe based on three-component force sensor for characterizing and quantifying surface roughness is proposed by using silicon micromachining technology. The sensor design is based on a cross-beam structure, which ensures that the system possesses high sensitivity and low coupling. The results show that the proposed sensor possesses high sensitivity, low coupling error, and temperature compensation function. The proposed system can be used to investigate micromechanical structures with nanometer accuracy.
\end{abstract}

Keywords: Surface roughness metrology, multi-component force sensor, Finite element analysis.

\section{INTRODUCTION}

$\mathrm{S}$ URFACE ROUGHNESS affects the function of how an object will interact with its environment, and it is becoming more important in industry as there is continuing need to solve the trade-off between manufacturing cost of component and its performance. A large number of research teams have presented their work on surface roughness metrology by employing available techniques such as AFM (Atomic Force Microscope), SEM (Scanning Electron Microscopy), and TEM (Transmission Electron Microscopy) [1]. Conveniently, the measurement methods for surface texture can be divided into two categories, i.e. contact and non-contact approaches. The contact measuring approach refers to detecting and quantifying roughness by using stylus based instruments, while the non-contact measuring approach means that the roughness is estimated by optical measurement instruments. Main advantages of the contact approach include structural simplicity, immunity to covering oil film and optical properties of object, and the most common contact approach to roughness measurement is based on AFM. MEMS (Micro-Electromechanical Systems) devices benefit from miniaturization, low power, low cost, low weight, high performance, easy mass-production and integration, and they have evolved from academic exercises to many crucial integral components such as automotive electronics, medical equipment and smart portable electronics over the past two decades [2].

As an important objective in the fields of material characterization, material science and micro-systems testing, micromechanical multi-component force sensor is used to measure force and moment information when the size range of objects manipulation is from microns to nanometers [3]. In past years micromechanical force sensors have been developed to perform characterization of micromechanical structures, such as determination of their spring characteristics and quantifying the surface roughness [4], [5], which cannot be accomplished by conventional force sensors due to their size, resolution and measurement range. Gao et al. [6] used a micromechanical probe-measuring instrument to estimate the surface properties and characterize in situ surface topography. A load-adding cantilever and force-sensing cantilever were used for surface indentation and profile measurement to obtain the loaddepth plot and scanning image.

The optical approach has been successfully utilized in metrology due to its high measurement velocity and noncontact measurement mode. But its application is restricted to specimen with low aspect ratio, i.e. free of features such as vertical sidewalls, undercuts and deep narrow drillings [7], [8]. Besides, the determination of three-component force vector is necessary for characterizing and quantifying surface roughness of complex specimen.

In this paper, a novel micromechanical three-component force sensor with cross-beam structure based on measurement of piezoresistor that occurred on force-sensing element is proposed. Particular attention is devoted to measuring principle, analysis and characterization of the proposed sensor. The sensor is designed to measure the normal force $F_{z}$ and two tangential force terms $F_{x}, F_{y}$. With those revealed three component force information, the surface roughness can be characterized and quantified rapidly.

\section{DESIGN OF THE SENSOR}

The most critical mechanical component of almost all kinds of force sensors is Elastic Element (EE), which serves as reaction mechanism to applied forces/moments by its deformation or strain [9]. Some of the most important design criteria are as follows [2], [10]: (1) Global Sensitivity 
and Minimum Stiffness, (2) Simple Minimum Volume, Especially Reduced Height, (3) Measurement Isotropy and Low Coupling Effects among Components, and (4) Small Error of Nonlinearity, Hysteresis and Repeatability. In this paper, a sophisticated EE structure is proposed based on cross-beams to fulfill the above criteria.

The micromechanical three-component force sensor based on cross-beams, as shown in Fig.1., is composed of base, a stylus standing on a central platform that serves as connection with the objects, and four radial spoke portions that extend outwardly from the platform to the base. The four cross beams with a rectangular parallelepiped shape (with a size of $400 \times 100 \times 20 \mu^{3}$ ) play the role of active sensing portions sensitive to normal force $\left(F_{z}\right)$ and both tangential force terms $\left(F_{\mathrm{x}}, F_{\mathrm{y}}\right)$. In the case of measurement, the annular rim is fixed as a base frame and the platform is applied with forces and moments. The overall dimensions of the sensor are $2400 \times 2400 \times 700 \mu \mathrm{m}^{3}$. The sensor could be fabricated by anisotropic wet chemical etching using a doublesided silicon ( 100 l 0 ) wafer via standard CMOS technology [11]. 12 piezoresistors are fabricated via standard processing steps for insulation, diffusion and metallization on the cross-beams. The measuring force applied to the top of the stylus will result in cross-beam deformation, and the piezoresistors mounted on the crossbeams will undergo the changes of resistances that are proportional to the applied measuring force. The strain $\varepsilon_{x}$ and stress $\sigma_{x}$ occupied on the upper surface $(z=h / 2)$ of the beams can be obtained as follows

$$
\begin{gathered}
\varepsilon_{x}=-\frac{\partial_{\omega}^{2}}{\partial x^{2}} z=\frac{F h}{2 E J}\left[L-\left(x-L_{h}\right)\right] \\
\sigma_{x}=-E \frac{\partial_{\omega}^{2}}{\partial x^{2}} z=\frac{F h}{2 J}\left[L-\left(x-L_{h}\right)\right]
\end{gathered}
$$

where $L_{h}=1 / 2 L$, cross sectional moment of inertia $J=b h^{3} / 12$, and $b, h$ and $L$ are width, thickness and length of the beam.

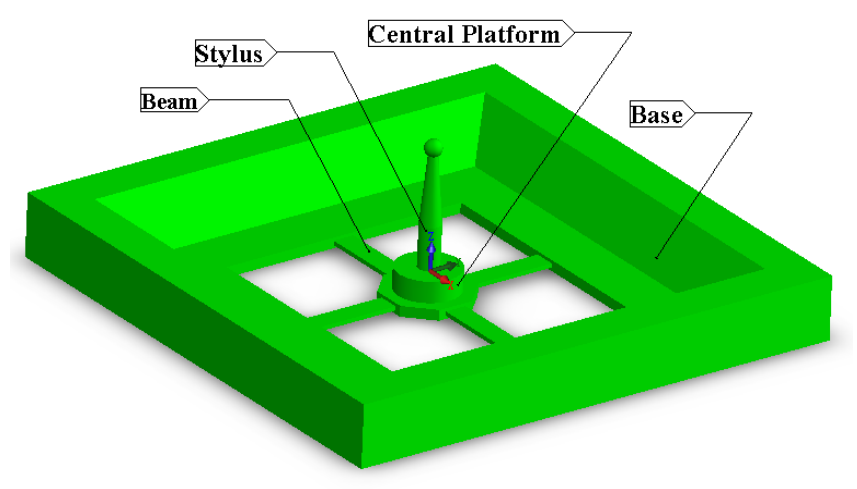

Fig.1. A perspective view of the proposed micromechanical threecomponent force sensor based on cross-beams.

\section{PRINCIPLE OF MEASUREMENT AND FINITE ELEMENT ANALYSIS}

Numerous force sensing technologies are available, e.g. capacitive, inductive, piezoelectric, piezoresistive, magnetic, and optical methods [12]. Among them, the most common method for detecting force is the piezoresistive approach, which always adopts a piezoresistor as an electric measurement element [13], [14], [15]. The measurement force is translated into a voltage by the resistance change in piezoresistors that are bonded on the EE of the force sensor. The piezoresistors can be formed by doping a silicon beam along central-longitudinal axes on upper surface of the cross-beam or from deposited polysilicon or metal layers.

According to the piezoresistance effect, the relative change of resistance of conventional piezoresistor having area $A_{\mathrm{p}}$ is defined as [16].

$\frac{\Delta R}{R}=\frac{1}{A_{p}} \int_{A_{p}}\left(\chi_{11}^{\prime} \sigma_{1}+\chi_{12}^{\prime} \sigma_{2}+\chi_{13}^{\prime} \sigma_{3}+\chi_{14}^{\prime} \tau_{4}+\chi_{15} \tau_{5}+\chi_{16}^{\prime} \tau_{6}\right) d A(3)$

where the primed quantities $\chi_{1 i}(i=1,2,3,4,5,6)$ are the piezoresistance coefficients referred to arbitrarily oriented axes, and $\sigma_{j}(j=1,2,3)$ and $\tau_{k}(k=4,5,6)$ represent the normal stresses and shear stresses.

The output voltage variation is proportional to relative resistance variation of Wheatstone bridge,

$$
\Delta U=\frac{U_{E}}{4}\left(\frac{\Delta R_{1}}{R_{1}}-\frac{\Delta R_{2}}{R_{2}}+\frac{\Delta R_{3}}{R_{3}}-\frac{\Delta R_{4}}{R_{4}}\right)
$$

where $R_{i}$ and $\Delta R_{i}, i=1,2,3,4$, represent the resistance and its variation of the $i$ th piezoresistor, respectively.

The relationship between measuring force $\mathrm{F}=\left(F_{x}, F_{\mathrm{y}}, F_{\mathrm{z}}\right)^{\mathrm{T}}$ which contains the forces $F_{x}, F_{\mathrm{y}}$ and $F_{\mathrm{z}}$ in $x, y$, and $z$ directions and outputs of Wheatstone bridge can be expressed as follows by using detection matrix $\mathrm{T}$ :

$$
\mathrm{S}=\mathrm{TF}
$$

Accordingly, from the sensor outputs and based on the decoupling method, the applied forces and moments can be determined and calculated as follows:

$$
\mathrm{F}=\mathrm{T}^{-1} \mathrm{~S}
$$

When $\mathrm{S}$ contains more than three elements, the Moore Penrose inverse techniques must be employed to determine the measuring force $\mathrm{F}$ from (5) as

$$
F=\left(T^{T} T\right)^{-1} \times T^{T} S
$$

The approach of Finite Element Analysis (FEA) provided by the software ANSYS $^{\circledR}$ is used to help understand the static characteristics and dynamic behavior of the EE structure and determine the appropriate positions and arrangement to bond the strain gauges. A three dimensional 
solid model of the sensor is meshed using higher order, ten node tetrahedral structural elements, SOLID187, supported by the software ANSYS ${ }^{\circledR}$ (see Fig.2.). The loads and support are applied to the top of the stylus and the base of the sensor, respectively.

The deformation of the stylus top can be calculated by

$$
\delta=\left[\begin{array}{l}
\delta_{x} \\
\delta_{y} \\
\delta_{z}
\end{array}\right]=\mathrm{kF}=\left[\begin{array}{ccc}
k_{x x} & 0 & 0 \\
0 & k_{y y} & 0 \\
k_{x z} & k_{y z} & k_{z z}
\end{array}\right]\left[\begin{array}{l}
F_{x} \\
F_{y} \\
F_{z}
\end{array}\right]
$$

where $\delta$ and $\mathrm{k}$ represent the deformation vector and stiffness matrix, respectively, which can be easily derived from mechanics of materials.

When the specimen is moved by a precision stage, a feedback loop is used to maintain the excursion of stylus tip at a constant level with the specimen. Therefore, the surface topography of specimen can be obtained by variation of deformation.

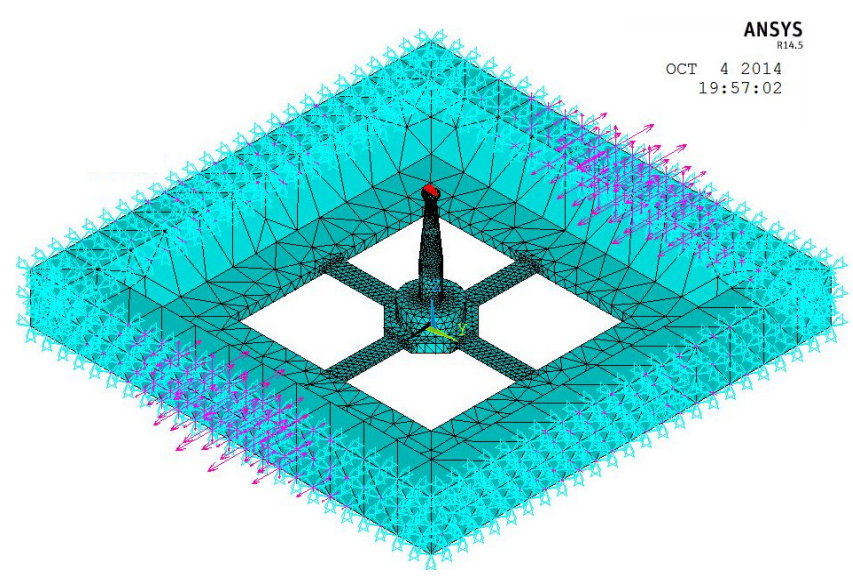

Fig.2. Sensor model meshed with SOLID187 elements.

\subsection{Under applied measuring force component $F_{z}$}

The FEA (Finite Element Analysis) results of the physical deformations, distributions of stress and strain due to force along Z-axis with amplitude of $0.01 \mathrm{~N}$ are shown in Fig.3.

In the strain and stress distribution figures, the dark blue denotes the negative direction strain or stress while light blue indicates positive direction strain or stress, and the color depth illustrates the strain value. The stress and strain are concentrated near the platform and base of the sensor, and the patterns of their distributions are similar to those predicted by the theory mentioned above.

Because of the Z-axial symmetry of load and structure, all four beams have the same response, and the maximum deformation, maximum equivalent stress and maximum strain occurring on the cross-beam are $1.206 \mu \mathrm{m}$, 83.7593 $\mathrm{MPa}$ and $0.000446 \mathrm{~mm} / \mathrm{mm}$, respectively.Fig.4. shows the Path diagram of stress along the cross-beam.

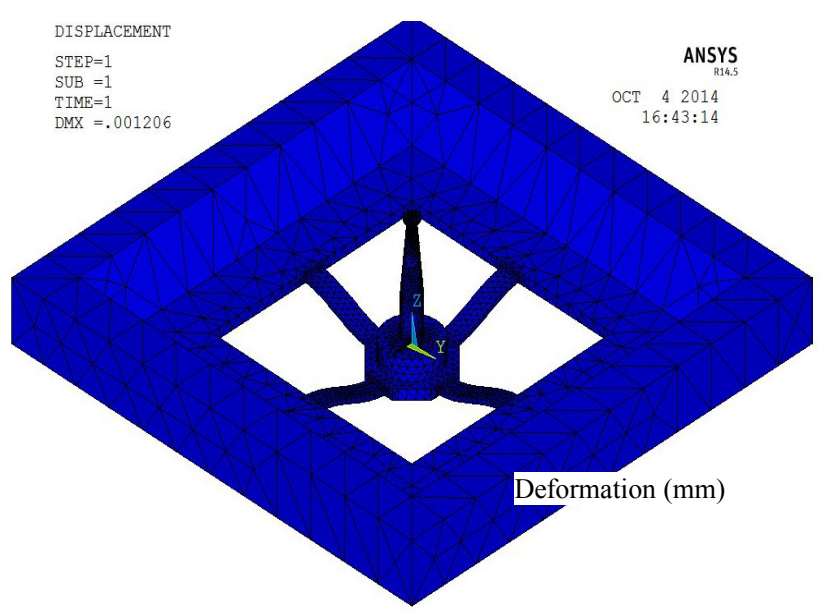

(a)

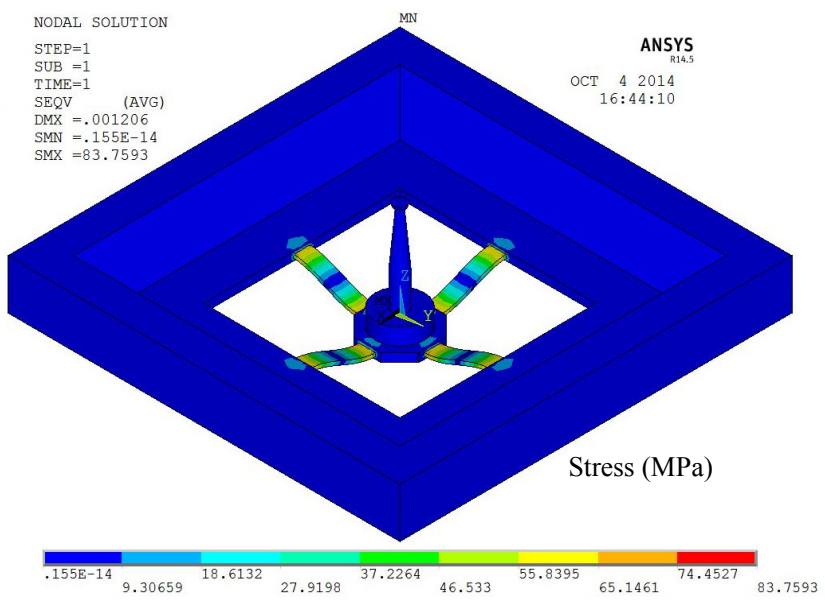

(b)

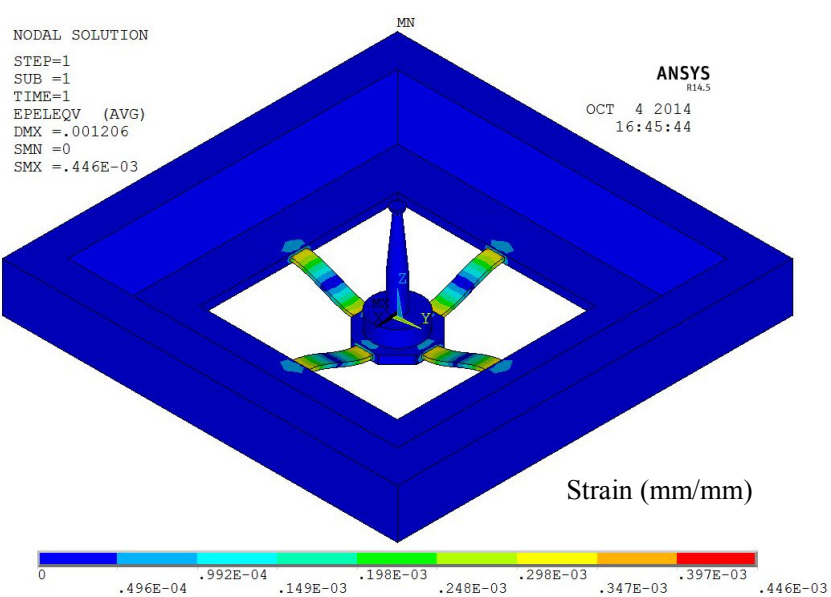

(c)

Fig.3. FEA results due to $F_{z}(0.01 \mathrm{~N})$ : (a) total deformation, (b) stress distribution, and (c) strain distribution. 


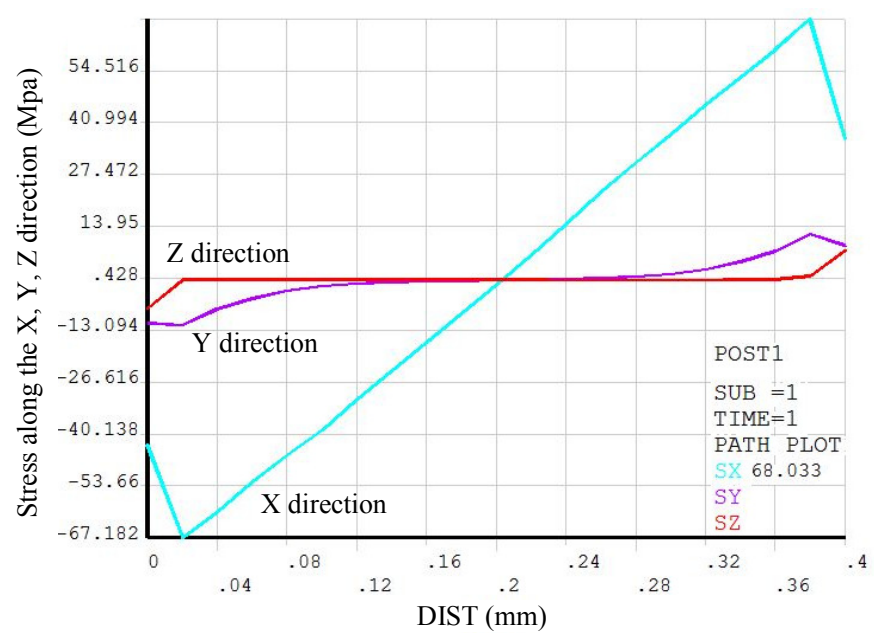

(a)

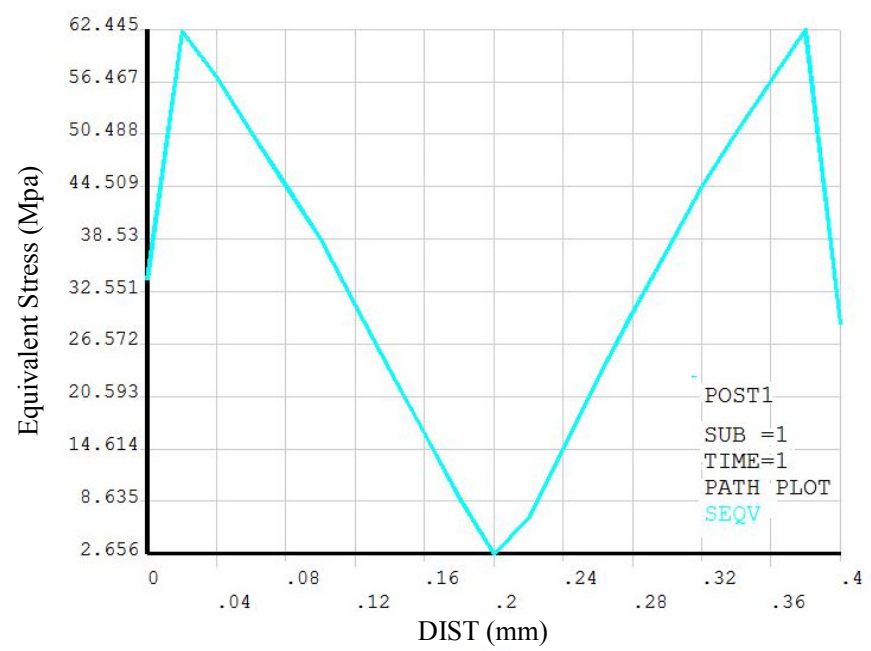

(b)

Fig.4. Path diagram of stress $(\mathrm{MPa})$ due to the $F_{z}(0.01 \mathrm{~N})$ along cross-beam (a) three-dimensional stress (b) equivalent stress.

\subsection{Under applied measuring force component $F_{x}$}

The FEA results of the physical deformations, distributions of stress and strain due to force along $\mathrm{x}$-axis with amplitude of $0.01 \mathrm{~N}$ are shown in Fig.5. The main stress and strain occur on the cross-beam along the $\mathrm{X}$-axis (beam 1 and 3). When the elastic element is applied with measuring force $F_{y}$, the result is similar.

It is observed that the stress and strain occurring on beams 1 and 3 are distributed along the $\mathrm{X}$-axis direction and reach the positive and negative maximum values near the central platform, and the second maximum values arise near the base of sensor. The strain and stress along the Y-axis direction is weak. The maximum deformation, maximum equivalent stress and maximum strain occurring on the cross-beam are $\quad 7.132 \mu \mathrm{m}, \quad 261.989 \mathrm{MPa}$ and $0.001395 \mathrm{~mm} / \mathrm{mm}$, respectively. Fig.6. shows the Path diagram of stress due to $F_{x}(0.01 \mathrm{~N})$ along cross-beams 1 and 2 .

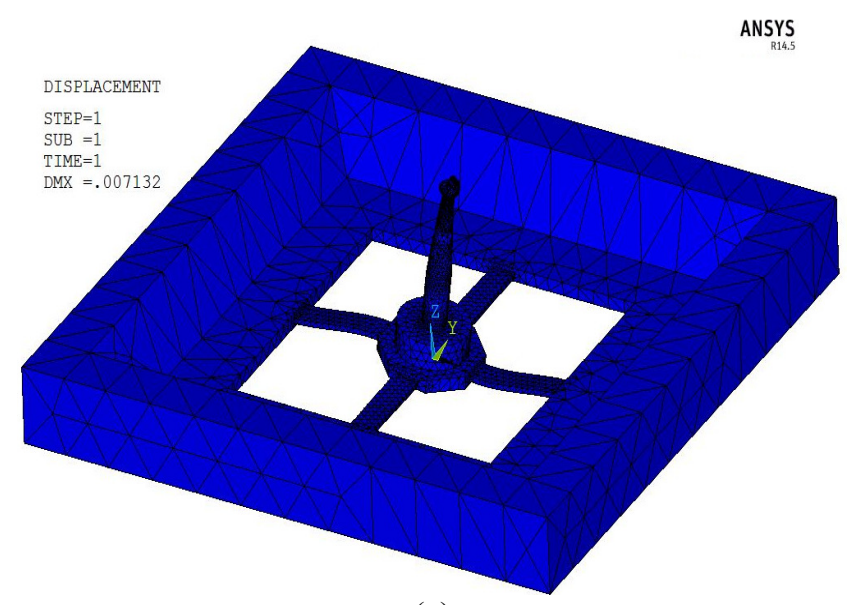

(a)

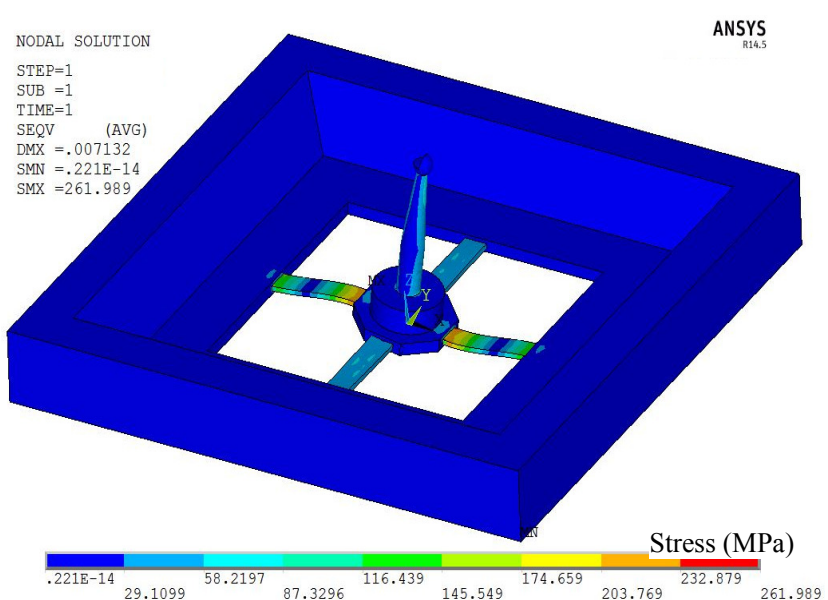

(b)

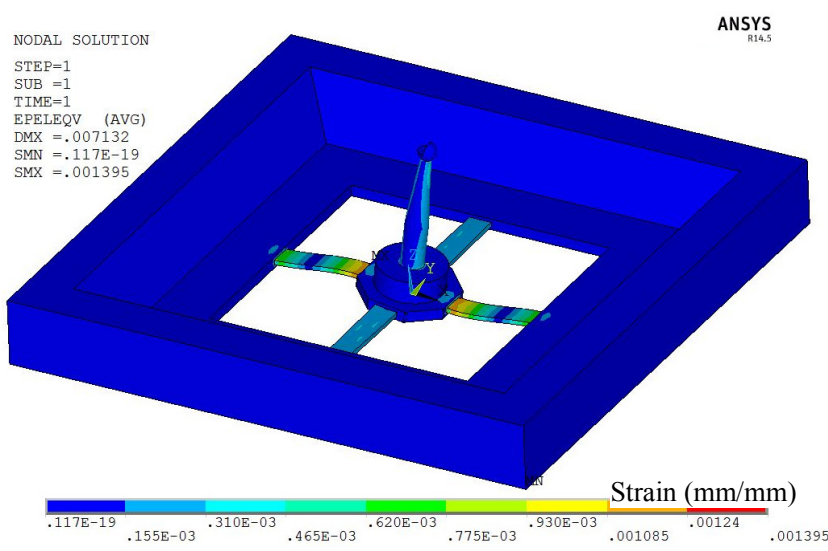

(c)

Fig.5. FEA results due to the $F_{x}(0.01 \mathrm{~N})$ : (a) total deformation, (b) stress distribution, and (c) strain distribution. 


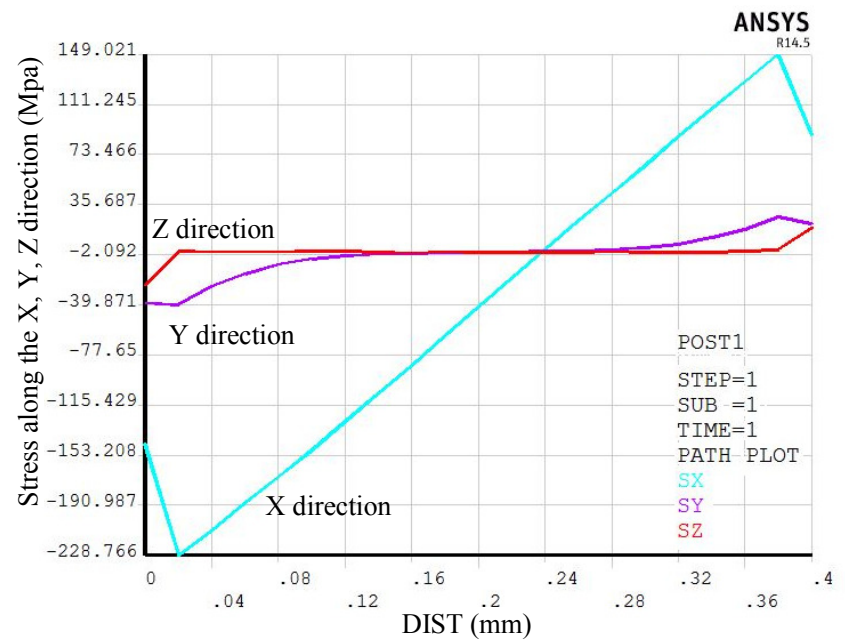

(a)

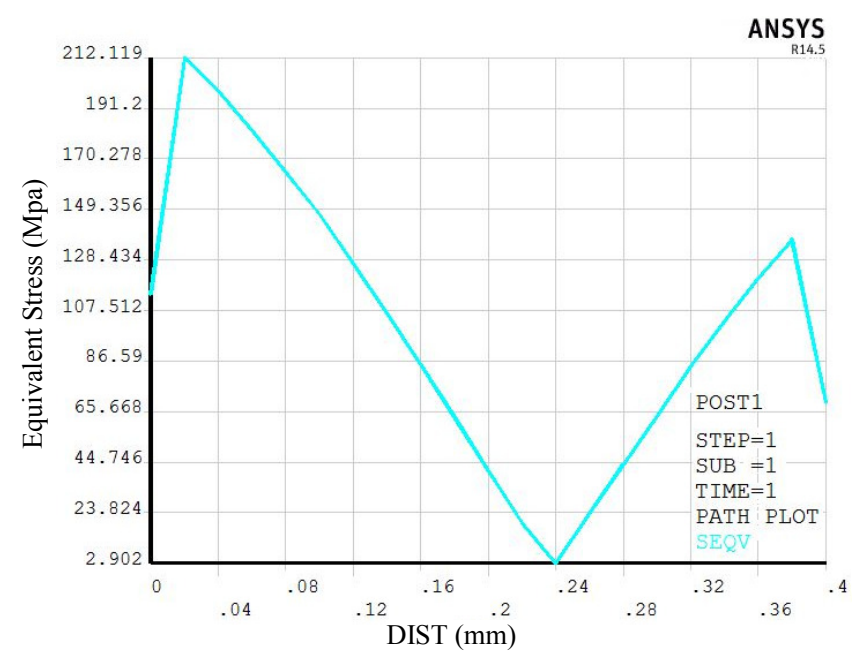

(b)

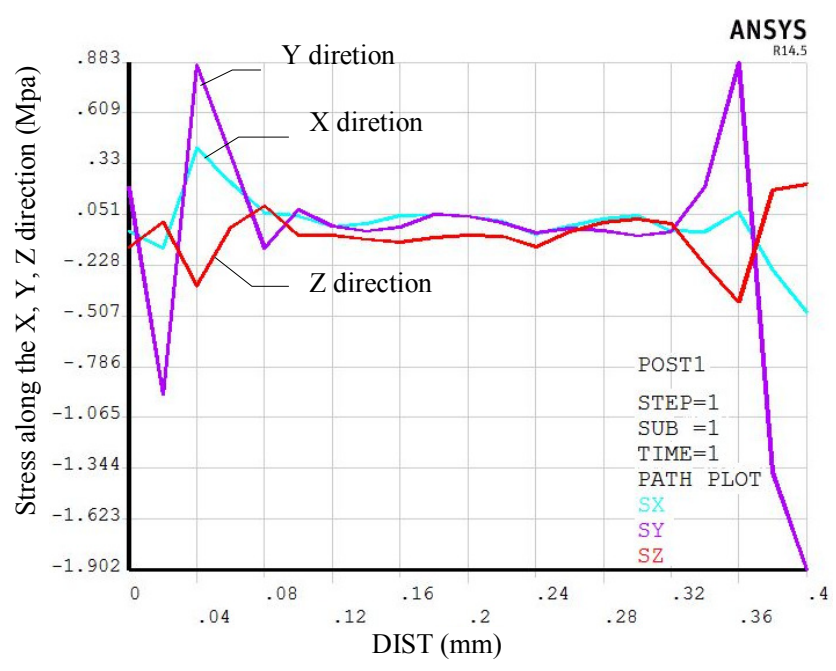

(c)

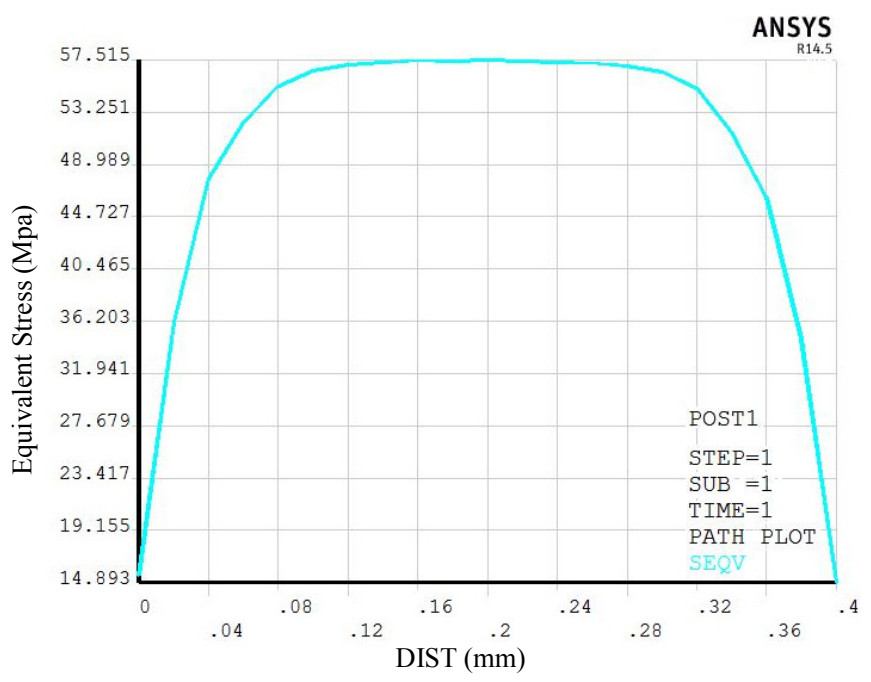

(d)

Fig.6. Path diagram of stress $(\mathrm{MPa})$ due to $F_{x}(0.01 \mathrm{~N})$ (a) threedimensional stress along cross-beam 1 (b) equivalent stress along cross-beam 1(c) three-dimensional stress along cross-beam 2 (d) equivalent stress along cross-beam 2 .

\subsection{Dynamic analysis}

The sensor will behave dynamically when it is subject to applied measuring force. Here a modal analysis via ANSYS that confirms the sensor has good dynamic performance, e.g. vibration characteristics is performed. A fixed support is applied to the base of sensor, and the number of frequencies of interest is specified to first six natural frequencies. The first six mode shapes of sensor are shown in Fig.7. The first six natural frequencies are also obtained via the software, as listed in Table 1. 
In tapping mode, the stylus is driven at the resonant frequency of fundamental mode with the tip periodically tapping on the surface. In order to enhance the signal level at a given harmonics of fundamental mode, a harmonic analysis via ANSYS is performed.

Table 1. The first six natural frequencies.

\begin{tabular}{ccccccc}
\hline Mode & $\mathbf{1}$ & $\mathbf{2}$ & $\mathbf{3}$ & $\mathbf{4}$ & $\mathbf{5}$ & $\mathbf{6}$ \\
\hline Natural & 1648. & 2605. & 2612. & 1653 & 1678 & 2202 \\
Frequenc & 2 & 3 & 0 & 4 & 4 & 7 \\
y (Hz) & & & & & & \\
\hline
\end{tabular}

The harmonic analysis results are plotted in Fig.8. As it is shown, the natural frequencies for measuring force component $F_{z}$ and $F_{x}$ are $980 \mathrm{~Hz}$ and $1038 \mathrm{~Hz}$, respectively.

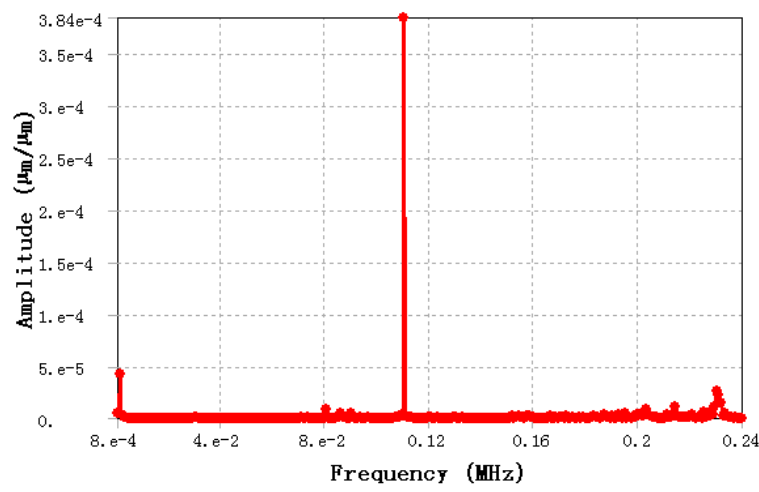

(a)

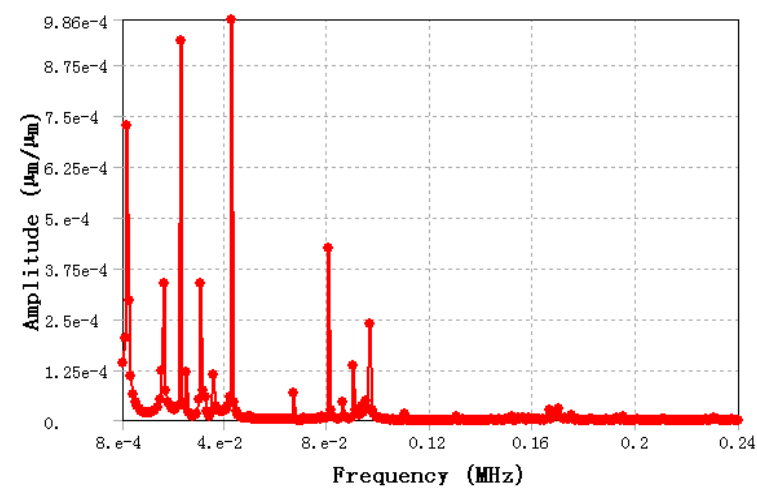

(b)

Fig.8. Harmonic response of the cross-beam under the measuring force component $F_{z}$ (a) and $F_{x}(\mathrm{~b})$.

\section{ARRANGEMENT OF PIEZORESISTORS AND MEASURING CIRCUITS}

It is important to place piezoresistors to proper place with appropriate orientation. The piezoresistors are normally placed at the pots that have maximum strains and along the direction of stress in order to obtain greater sensitivity, repeatability and higher-level output, as shown in Fig.9. In this study, the piezoresistors are bonded $0.2 \mu \mathrm{m}$ away from edges of the cross-beams and thus are uniformly strained when subjected to measuring forces.

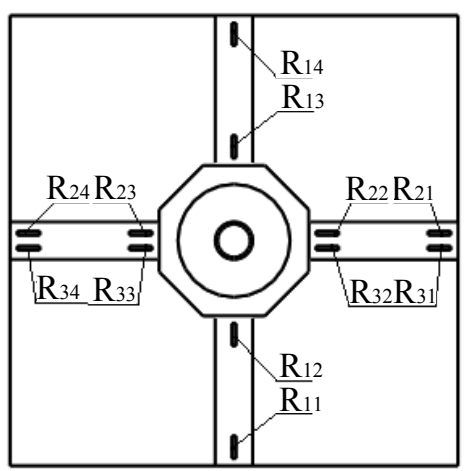

Fig.9. Arrangement of piezoresistors.

Wheatstone bridges are used to convert the resistance changes of piezoresistors to voltage output due to their high sensitivity, wide measurement range, high precision, and simple configuration, as shown in Fig.10. Group $\mathrm{Y}$ is composed of piezoresistors $\mathrm{R}_{11}, \mathrm{R}_{12}, \mathrm{R}_{13}$ and $\mathrm{R}_{14}$ that along the radial direction are arranged to detect measuring force component $F_{y}$. Also Group $\mathrm{X}$ is composed of piezoresistors $\mathrm{R}_{21}, \mathrm{R}_{22}, \mathrm{R}_{23}$ and $\mathrm{R}_{24}$ that along the radial direction are arranged to detect measuring force component $F_{x}$. Furthermore, Group $\mathrm{Z}$ is composed of piezoresistors $\mathrm{R}_{31}$, $\mathrm{R}_{32}, \mathrm{R}_{33}$ and $\mathrm{R}_{34}$ that along the radial direction are arranged to detect measuring force component $F_{z}$.

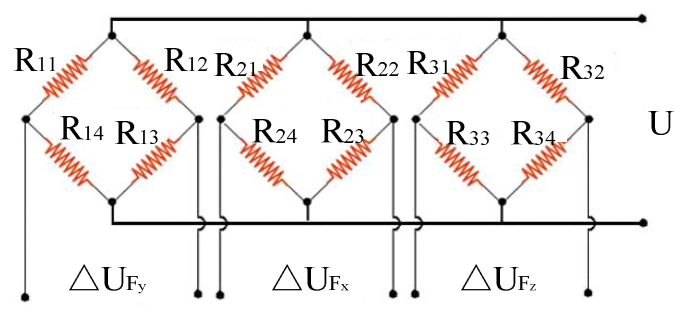

Fig.10. Wheatstone bridges connection for piezoresistors.

\section{RESULTS}

\subsection{Under measuring force component $F_{z}$}

As shown in Fig.3., Fig.4., Fig.9., and Fig.10., when the sensor bears the measuring force component $F_{z}$ and environment temperature changes, the resistances of piezoresistors will change correspondingly as follows:

$\frac{\Delta R_{11}}{R_{11}}=\frac{\Delta R_{14}}{R_{14}}=\frac{\Delta R_{21}}{R_{21}}=\frac{\Delta R_{24}}{R_{24}}=\frac{\Delta R_{31}}{R_{31}}=\frac{\Delta R_{34}}{R_{34}}=\left(\frac{\Delta R_{11}}{R_{11}}\right)_{\varepsilon}+\left(\frac{\Delta R}{R}\right)_{t}$

$\frac{\Delta R_{12}}{R_{12}}=\frac{\Delta R_{13}}{R_{13}}=\frac{\Delta R_{22}}{R_{22}}=\frac{\Delta R_{23}}{R_{23}}=\frac{\Delta R_{32}}{R_{32}}=\frac{\Delta R_{33}}{R_{33}}=\left(\frac{\Delta R_{12}}{R_{12}}\right)_{\varepsilon}+\left(\frac{\Delta R}{R}\right)_{t}$

Therefore, each bridge output voltage can be obtained as follows:

$$
\Delta U_{F x}=\frac{U}{4}\left(\frac{\Delta R_{21}}{R_{21}}-\frac{\Delta R_{22}}{R_{22}}+\frac{\Delta R_{23}}{R_{23}}-\frac{\Delta R_{24}}{R_{24}}\right)=0
$$




$$
\begin{aligned}
\Delta U_{F y} & =\frac{U}{4}\left(\frac{\Delta R_{11}}{R_{11}}-\frac{\Delta R_{12}}{R_{12}}+\frac{\Delta R_{13}}{R_{13}}-\frac{\Delta R_{14}}{R_{14}}\right)=0 \\
\Delta U_{F z} & =\frac{U}{4}\left(\frac{\Delta R_{31}}{R_{31}}-\frac{\Delta R_{32}}{R_{32}}+\frac{\Delta R_{34}}{R_{34}}-\frac{\Delta R_{33}}{R_{33}}\right) \\
& =\frac{U}{2}\left(\left(\frac{\Delta R_{31}}{R_{31}}\right)_{\varepsilon}-\left(\frac{\Delta R_{32}}{R_{32}}\right)_{\varepsilon}\right)
\end{aligned}
$$

According to this analysis, only the bridge circuit of group $\mathrm{Z}$ is relative to the component $F_{z}$ while the other bridge outputs are irrelevant.

\subsection{Under measuring force component $F_{y}$ (similar to the component $F_{x}$ )}

When the measuring force $F_{\mathrm{y}}$ is applied to stylus and environment temperature changes, strain and temperature will increase or decrease the resistances of piezoresistors as follows:

$$
\begin{gathered}
\frac{\Delta R_{21}}{R_{21}}=\frac{\Delta R_{24}}{R_{24}}=\frac{\Delta R_{31}}{R_{31}}=\frac{\Delta R_{34}}{R_{34}}=\left(\frac{\Delta R_{21}}{R_{21}}\right)_{\varepsilon}+\left(\frac{\Delta R}{R}\right)_{t} \\
\frac{\Delta R_{22}}{R_{22}}=\frac{\Delta R_{23}}{R_{23}}=\frac{\Delta R_{32}}{R_{32}}=\frac{\Delta R_{33}}{R_{33}}=\left(\frac{\Delta R_{22}}{R_{22}}\right)_{\varepsilon}+\left(\frac{\Delta R}{R}\right)_{t} \\
\frac{\Delta R_{11}}{R_{11}}=\left(\frac{\Delta R_{11}}{R_{11}}\right)_{\varepsilon}+\left(\frac{\Delta R}{R}\right)_{t} \\
\frac{\Delta R_{14}}{R_{14}}=-\left(\frac{\Delta R_{11}}{R_{11}}\right)_{\varepsilon}+\left(\frac{\Delta R}{R}\right)_{t} \\
\frac{\Delta R_{13}}{R_{13}}=\left(\frac{\Delta R_{13}}{R_{13}}\right)_{\varepsilon}+\left(\frac{\Delta R}{R}\right)_{t} \\
\frac{\Delta R_{12}}{R_{12}}=-\left(\frac{\Delta R_{13}}{R_{13}}\right)_{\varepsilon}+\left(\frac{\Delta R}{R}\right)_{t}
\end{gathered}
$$

where $\left(\Delta R_{i} / R_{i}\right)_{\varepsilon}$ and $(\Delta R / R)_{t}$ indicate the changed rate of resistance for piezoresistor $R_{\mathrm{i}}$ and temperature variation, respectively.

Therefore, each bridge output voltage can be obtained as follows:

$$
\begin{aligned}
\Delta U_{F x} & =\frac{U}{4}\left(\frac{\Delta R_{21}}{R_{21}}-\frac{\Delta R_{22}}{R_{22}}+\frac{\Delta R_{23}}{R_{23}}-\frac{\Delta R_{24}}{R_{24}}\right) \\
& =\frac{U}{2}\left(\left(\frac{\Delta R_{21}}{R_{21}}\right)_{\varepsilon}-\left(\frac{\Delta R_{22}}{R_{22}}\right)_{\varepsilon}\right) \approx 0
\end{aligned}
$$

$$
\begin{aligned}
\Delta U_{F y} & =\frac{U}{4}\left(\frac{\Delta R_{11}}{R_{11}}-\frac{\Delta R_{12}}{R_{12}}+\frac{\Delta R_{13}}{R_{13}}-\frac{\Delta R_{14}}{R_{14}}\right) \\
& =\frac{U}{2}\left(\left(\frac{\Delta R_{11}}{R_{11}}\right)_{\varepsilon}+\left(\frac{\Delta R_{13}}{R_{13}}\right)_{\varepsilon}\right) \\
\Delta U_{F z} & =\frac{U}{4}\left(\frac{\Delta R_{31}}{R_{31}}-\frac{\Delta R_{32}}{R_{32}}+\frac{\Delta R_{34}}{R_{34}}-\frac{\Delta R_{33}}{R_{33}}\right) \\
& =\frac{U}{2}\left(\left(\frac{\Delta R_{31}}{R_{31}}\right)_{\varepsilon}-\left(\frac{\Delta R_{32}}{R_{32}}\right)_{\varepsilon}\right) \approx 0
\end{aligned}
$$

According to this analysis, only the bridge circuit of group $\mathrm{Y}$ is relative to the component $F_{y}$ while the other bridge outputs are irrelevant.

In addition, the sensor contains the hardware that can stabilize sensitivity over temperature. Specifically, this compensation method optimizes the accuracy over a range of $-50{ }^{\circ} \mathrm{C}$ to $+80{ }^{\circ} \mathrm{C}$ [17], [18].

\section{CONCLUSIONS}

A piezoresistive three-component force sensor intended for characterizing and quantifying surface roughness is proposed. A sophisticated EE structure based on crossbeams is adapted to fulfill the specific criteria such as high sensitivity, simple structure, and low anisotropic and coupling nonlinearity. In order to find the optimum position of piezoresistors, the strain, stress and deformation responses at the specified positions are formulated through the Finite Element method. 12 piezoresistors are connected in three full-bridge configurations with each bridge output representing a measuring force component. The results show that the proposed sensor possesses high sensitivity and low coupling error. With those revealed force information, surface patterns and mechanical properties, such as softness, friction, mechanical impedance, spring characteristics of micromechanical structures, and micro force exerted by a micro-actuator, can be characterized simultaneously and rapidly.

\section{ACKNOWLEDGMENT}

This work is supported in part by the National Nature Science Foundation of China (NSFC 61203207), specialized Research Fund for the Doctoral Program of Higher Education (No. 20120161120015, No.20130161110009), the Young Core Instructor Foundation of Hunan Provincial Institutions of Higher Education, Hunan provincial natural science foundation of China (14JJ1011), and the Changsha science and technology program (k1501009-11).

\section{REFERENCES}

[1] Thomas, T.R. (1998). Trends in surface roughness. International Journal of Machine Tools and Manufacture, 38 (5), 405-411. 
[2] Liang, Q., Zhang, D., Coppola, D., Wang, Y., Sun, W., Ge, Y. (2014). Multi-dimensional MEMS/micro sensor for force and moment sensing: A review. IEEE Sensors Journal, 14 (8), 2643-2657.

[3] Beyeler, F., Muntwyler, S., Nelson, B.J. (2009). A sixaxis MEMS force-torque sensor with micro-Newton and nano-Newtonmeter resolution. IEEE Journal of Microelectromechanical Systems, 18 (2), 433-441.

[4] Bütefisch, S., Büttgenbach, S., Kleine-Besten, T., Brand, U. (2001). Micromechanical three-axial tactile force sensor for micromaterial characterization. Microsystem Technologies, 7 (4), 171-174.

[5] Saif, M.T.A., MacDonald, N.C. (1998). Measurement of forces and spring constants of micro-instruments. Review of Scientific Instruments, 69 (3), 1410-1422.

[6] Gao, P., Yuan, Z. (1999). Development of a micromechanical probe-measuring instrument for surface properties characterization. Measurement Science and Technology, 10 (8), 105-108.

[7] Ruther, P. et al. (2005). Novel 3D piezoresistive silicon force sensor for dimensional metrology of micro components. In 2005 IEEE Sensors, Oct. 30 Nov. 3, 2005. IEEE.

[8] Shen, Y., Wang, Y., Zaklit, J. (2010). Development of an optical surface characterization sensor for simultaneously measuring both 3-D surface texture and mechanical properties. In 2010 IEEE Sensors, November 1-4, 2010. IEEE, 1892-1895.

[9] Wilson, J.S. (2004). Sensors Technology Handbook. Elsevier.

[10] Yin, Y., Zhou, C., Chen, S., Hu, H., Lin, Z. (2006). Optimal design of micro-force sensor for wire bonding with high acceleration and frequent movement. Sensors and Actuators A: Physical, 127 (1), 104-118.
[11] Dauer, S., Buttgenbach, S., Ehlert, A. (1998). Rapid prototyping of micromechanical devices using a Qswitched Nd: YAG laser with optional frequency doubling. In Eurosensors '98, September 1998, 7-10.

[12] Ştefãnescu, D.M. (2011). Handbook of Force Transducers: Principles and Components. Springer.

[13] Jia, W., Porta, M., Tichem, M., Staufer, U., Sarro, P.M. (2013). Integrated piezoresistive force and position detection sensors for micro-handling applications. Journal of Microelectromechanical Systems, 22 (6), 1310-1326.

[14] Jiang, Y.G., Ono, T., Esashi, M. (2008). Fabrication of piezoresistive nanocantilevers for ultra-sensitive force detection. Measurement Science and Technology, 19 (8), 084011.

[15] Lu, Z., Luo, H., Chen, P.C., Lin, W. (2006). An integrated probe sensor for micro-force measurement. Measurement Science and Technology, 17 (4), 869.

[16] Pfann, W.G., Thurston, R.N. (1961). Semiconducting stress transducers utilizing the transverse and shear piezoresistance effects. Journal of Applied Physics, 32 (10), 2008-2019.

[17] Liang, Q., Zhang, D., Wang, Y., Ge, Y. (2013). Design and analysis of a novel six-component $\mathrm{F} / \mathrm{T}$ sensor based on CPM for passive compliant assembly. Measurement Science Review, 13 (5), 253-264.

[18] Fahlbusch, S., Fatikow, S. (1998). Force sensing in microrobotic systems-an overview. In 1998 IEEE International Conference on Electronics, Circuits and Systems. IEEE, Vol. 3, 259-262.

Received February 04, 2015. Accepted September 30, 2015. 